\title{
La alfabetización académica universitaria a examen: una propuesta para leer $y$ dialogar con el mundo ${ }^{12}$
}

\author{
University Academic Literacy under Review: a Proposal to Read \\ and Discuss with the World
}

CINTIA CARREIRA ZAFRA

MARCIN KAZMIERCZAK

MARÍA TERESA SIGNES

Universitat Abat Oliba CEU

España

ccarreiraz@uao.es

kaz@uao.es

msigness6o@uao.es

(Recibido: $15-\mathrm{O} 4-2 \mathrm{O} 2 \mathrm{O}$ aceptado: 2I-IO-2O2O)

Resumen. Las prácticas letradas contemporáneas indican que nos enfrentamos a nuevos retos: leer y escribir en el siglo XXI dista mucho de lo que se entendía de estas actividades en siglos pasados. Este cambio revierte también en las competencias que, desde el ámbito universitario, se tiene como objetivo desarrollar en los estudiantes. En la presente investigación se relata una experiencia pedagógica de promoción de la alfabetización académica llevada a cabo en la Universitat Abat Oliba CEU durante el curso 2017-2018 en el marco de un proyecto de innovación y mejora de la calidad docente. Consiste en la creación de una revista estudiantil "Letras de Batalla: debate académico actual": se describen las motivaciones y objetivos del proyecto, su metodología y fases de implementación. Por último, se detalla la relación entre resultados esperados y resultados obtenidos al finalizar la edición del primer número, publicado online y con ISSN propio. Así, la revista nos permite comprobar que, mediante la producción de artículos académicos, los jóvenes mejoran sus competencias lectoras y asumen con naturalidad estas prácticas letradas, acrecentando asimismo sus capacidades para entender el mundo que les rodea y dialogar con él, integrarse en la sociedad y ser competencialmente eficaces.

Palabras clave: prácticas letradas contemporáneas; alfabetización académica; literacidad crítica; comunicación multimodal; educación superior.

\footnotetext{
I Para citar este artículo: Carreira Zafra, Cintia; Kazmierczak, Marcin y Signes, Ma Teresa (2O2I). La alfabetización académica universitaria examen: una propuesta para leer dialogar con el mundo. Alabe 23 [www.revistaalabe.com] DOI: IO.15645/Alabe2O2I.23.7

${ }^{2}$ Esta investigación forma parte del proyecto ANDREIA «Fomento de la resiliencia en la educación primaria: innovación y formación continua del profesorado» (PID2OI9-IIIO32RB-Ioo) financiado por el Ministerio de Ciencia e Innovación de España y desarrollado por el grupo de investigación TRIVIUM «Familia, educación y escuela inclusiva».
} 
Abstract. Contemporary literacy practices indicate that we face new challenges: reading and writing in the 2 Ist century is far from what was understood about these activities in past centuries. This change also reverts to the competencies that, from the university level, are expected to be developed by students. In this research a pedagogical experience of promoting academic literacy is reported, and carried out at the Abat Oliba CEU University during the 2017-20I8 academic year in the framework of a project of innovation and improvement of teaching quality. It consists of the creation of a student's journal "Letras de Batalla: debate académico actual": the motivations and objectives of the project, its methodology and implementation phases, are described. Finally, it the relationship between expected results and results obtained at the end of the edition of the first issue is detailed, published online and with its own ISSN. Thus, the journal allows us to verify that, through the production of academic articles, students improve their reading skills and naturally assume these literacy practices while also increasing their abilities to understand and engage with the world around them and master the skills necessary for integration into society.

Keywords: contemporary literacy practices; academic literacy; critical literacy; multimodal communication; higher education. 


\section{Introducción}

Determinar que la realidad ha cambiado no parece, a primera vista, un asunto de reciente actualidad. Este cambio puede constatarse en todas las esferas de la vida sin ápice de dudas. Ahora bien, que ya sepamos que el mundo no es lo que era no significa que haya parado de cambiar y que se mantenga estable, sino más bien al contrario: la sociedad postmoderna, esta sociedad de la información y comunicación, avanza cada día más a pasos agigantados e influye también en la manera en que la realidad se representa y se problematiza, trascendiendo los modos tradicionales de expresión como lo eran hasta hace poco la lectura y la escritura ${ }^{3}$. Leer no es lo que era: desde enviar emails formales a desconocidos, navegar por internet para comprar un billete low cost a París, comprar un yogurt con bífidus pero o\% materia grasa o comprobar los efectos secundarios de una medicina. Se desvela aquí el verdadero significado de la lectura contemporánea: leer desde la comunidad, una acción que ya no implica solamente oralizar -es decir, producir sonido en voz alta a unas letras escritas en papel- sino mucho más (Cassany, 2006).

La corriente que lleva por nombre New Literacy Studies (o NEL, Nuevos Estudios de Literacidad) ha impuesto un giro a la manera en que hasta la década de los ochenta se había entendido la noción de literacidad ${ }^{4}$. Uno de sus referentes principales es Brian Street (2004), de quien destacamos el conciso y claro ensayo Cross-Cultural Approaches to Literacy. Desde que "el pensamiento se empezó a concebir como insertado en el funcionamiento social de prácticas letradas" (Zavala, Niño-Murcia y Ames, 2004: 8) se viene ahondando en la contextualización de la literacidad en el marco social ${ }^{5}$ que la rodea. Mediante los NEL se despiertan nuevos interrogantes en relación a las implicaciones sociales de las prácticas letradas contemporáneas en el acto de descodificar y a los efectos que producen en la mente humana y en la conducta, entre otros muchos.

Por práctica letrada, a la que también podemos definir como proceso de lectoescritura textual, nos remitimos al consenso entre la literatura científica, que sostiene que esta es aquello que los grupos humanos hacen con la escritura ${ }^{6}$. Ya no hay una única manera de comprender y abordar la lectura y la escritura, sino que debemos comprender estas prácticas de expresión e intercambio comunicativo del ser humano desde un punto de vista holístico y global, entendiendo que los discursos recibidos y producidos se insertan en interacciones sociales más amplias. De hecho, a día de hoy, es por todos sabido que adquirir habilidades se relaciona directamente con las prácticas

\footnotetext{
3 Véase a Rodríguez (2013), Reale (2005) y Lipovetsky y Charles (20I4).

${ }^{4}$ Por cuestiones de extensión, no nos detendremos a definir la noción de literacidad, traducida literalmente del vocablo inglés literacy, si bien remitimos a la investigación de Tang y Danielsson (2OI8: I-II), que aportan un actualizado estado de la cuestión así como sus proyecciones de futuro.

5 En palabras de Zavala (2008: 7I), "la literacidad siempre implica una manera de usar la lectura y la escritura en el marco de un propósito social específico", lo cual, según afirma el mismo autor más recientemente, hace que concibamos el lenguaje "como una acción que es siempre parte de las prácticas sociales” (2018: 6I).

${ }^{6}$ Véase a Barton y Hamilton (I998) para ahondar en la relación entre literacidad y práctica social, quienes definen a la primera como una actividad situada en el espacio entre el pensamiento y el texto.
} 
socioculturales ${ }^{7}$ en las que estas habilidades se producen (Cassany, Sala y Hernández, 2008). Así pues, lo más característico de esto es la noción de "práctica”, puesto que implica que es una herramienta que nos permite mediar en esta cultura discursiva a la que pertenecemos.

Una de estas nuevas formas de leer y escribir contemporáneas es la del discurso especializado: las prácticas letradas académicas. Leer artículos de divulgación científica es una práctica que con el tiempo ha ido alcanzando un papel significativo en los contextos sociales ajenos a la academia en los que tiene lugar la lectura y la escritura. Esto es así sobre todo porque operamos mediante la lectura y la escritura diariamente y en cualquiera que sea el contexto en el que nos encontremos y también porque los trabajos desempeñados requieren cada vez más de especialistas de ámbitos muy concretos en lugar de generalistas. El conocimiento se clasifica y así también lo hace la escritura académica, organizada por disciplinas. Ahora bien: ¿dónde estriba la mayor dificultad cuando los jóvenes -e incluso de los adultos- se enfrentan a este tipo de discursos, ya sea cuando deben leerlos o, incluso peor, producirlos? El hecho de no ser especialistas en tal o cual ámbito concreto de conocimiento. Para intentar resolver esta dificultad, se asume, lógicamente, que la universidad sea el lugar desde el que promover el encuentro con la alfabetización académica. Este término ha circulado durante los últimos años refiriéndose a "la necesidad que tienen las instituciones educativas de desarrollar en los estudiantes habilidades para interpretar y/o producir los textos científicos y académicos” (Marín, 2006: 30).

Las salidas profesionales para las que se preparan los estudiantes que están en nuestras aulas universitarias van a exigirles un nivel alto de comprensión lectora y producción escrita sobre aquellos textos de su disciplina, luego es urgente que desde las universidades respondamos a estas futuras demandas. Si entendemos que la alfabetización implica habilidades cognitivas amplias y que va más allá de la acción de aprender a leer o descodificar textos, aquella referida al mundo académico exige mucho más; rellenar una solicitud para una beca implica ejercer el derecho de pedirla y conocer el formato y convenciones sociales preestablecidas para formularla, y los artículos científicos son también una producción cultural a partir de la cual entender el mundo, la propia cultura y las ajenas, y dialogar con ellas. Cassany y Morales (2009: IIо) coinciden con que el agente de cambio debe ser la universidad en tanto que "lugar donde iniciar el aprendizaje formal de los textos especializados de cada disciplina”, si bien difieren en los métodos, que son vistos como desordenados y poco explícitos.

Por otro lado, advertimos un salto en cuanto a la actitud con la que el ser humano se enfrenta a estos textos científicos: se pasa de la cultura popular a la participativa, matiz que introduce la noción de literacidad crítica, gracias a la que usamos y analizamos

\footnotetext{
7 La relevancia de las prácticas socioculturales es tal que se llega a admitir incluso que estas son la manera de "generar, comunicar y negociar contenido significativo con la mediación de textos codificados en contextos de participación" (Lanksear y Knoble, 2006: 64), siendo la generación, la comunicación y la negociación acciones que necesitan del contexto social para darse y cobrar sentido.
} 
las palabras con la intención de ejercer un mayor control sobre la información que emitimos y la que recibimos. Lo participativo tiene que ver con lo crítico, con la idea de que nuestras intervenciones sean significativas y fundamentadas sobre bases teóricas sólidas. Consecuentemente, uno de los objetivos de la universidad al fomentar la producción y lectura de textos científicos debe ser que sepan comprender los puntos de vista que reciban y, sobre todo, contrastarlos con los suyos propios. Para aprender a leer críticamente -es decir, a leer tras las líneas- se necesita de lo literal -a leer las líneas-y lo inferencial -a leer entre líneas-, que permitirán, primero, descodificar y a continuación inferir significados (Cassany, 20I9: I6).

Algunos psicólogos cognitivistas, en la década de los ochenta, enfatizaban la comprensión holística del texto más que los significados particulares y por ello la lectura en voz alta quedó relegada, para ellos, a un segundo plano. Efectivamente, es posible comprender la idea principal de un texto con solo unas pocas palabras, sin necesidad de pronunciar en voz alta. Esto es así si definimos la lectura como la adquisición e interiorización del significado de un texto a partir de indicios. Otro de los efectos que la contemporaneidad ha provocado en la lectura es el traslado del enfoque psicolingüístico al sociocultural: el mensaje es, además de una unidad cognitivo-lingüística, un artefacto sociopolítico, y con más razón todavía se erige la necesidad de fomentar la conciencia crítica de los universitarios al iniciarse en el debate académico.

En línea con la integración de nuevos métodos para la alfabetización académica que puedan convertirla en una práctica informal organizada y explícita dentro de las universidades, debemos tener en cuenta el potencial del entorno digital y, por ende, de la cultura del open-access que domina las redes. En esta "ciudad hipertextualizada" en la que leer el mundo y comunicarnos con él tiene muchas formas, imágenes y lenguajes -a saber el televisivo, cinematográfico, fotográfico, publicitario...-la lectura en el ciberespacio -esto es, una lectura veloz y discontinua por voluntad propia- es también una realidad (Kozak Rovero, 20oI), y no precisamente menos alejada de los jóvenes de lo que ya lo está la lectura tradicional sino más bien al contrario. No podemos olvidar que los universitarios de hoy, siguiendo la metáfora de Prensky (2OOI), son los nativos digitales que fluyen en la red como peces en el agua, mientras que nosotros somos los inmigrantes digitales, aquellos mamíferos que han lanzado al mar y deben aprender a nadar y sobrevivir en este nuevo medio a la par que enseñar a los peces que ya habitan en él. Los beneficios provocados por la multimodalidad de lo digital son muchos, ya que las posibilidades de referirse a la realidad son casi infinitas y creativas, y destacamos sobre todo el incremento exponencial de interlocutores a los que el texto se destina: el debate académico existe, está ahí fuera, y cualquiera puede participar -cuando no participa yaen él (Cassany, 2012).

\footnotetext{
${ }^{8}$ Muchos autores se han referido a la hipertextualización, de entre los que destacamos a Rodríguez (2OI3) por su reconfiguración de esta noción aplicada a la escuela contemporánea, paradigma por excelencia de lugar físico donde se aprende a leer la vida.
} 


\section{Objetivos}

La experiencia pedagógica que detallamos a continuación es fruto de la investigación llevada a cabo en el marco de la promoción de la alfabetización científica contextualizada previamente, y consiste en la implantación de una revista estudiantil universitaria en la realidad del campus, ampliando así la oferta de actividades extracurriculares al alcance de toda la comunidad universitaria.

La revista fue fundada en el año 2005 y hasta el año 2013 llegó a tener i6 números. En aquella primera etapa, el nombre completo de la revista era Letras de Batalla. Revista de literatura y cine de la Universitat Abat Oliba CEU. Los primeros I4 números fueron digitales, mientras que los últimos dos tuvieron tanto una versión digital como en papel. El nombre de Letras de Batalla fue elegido por los estudiantes para referirse a la batalla por salvaguardar la palabra escrita, puesto que, en su origen, la expresión se remonta al procedimiento medieval entre los caballeros que se retaban en duelo: cuando uno quería retar a otro, le entregaba una letra de batalla.

Por diversos motivos, el proyecto se detuvo hasta 2or8, cuando un grupo de investigadores - los autores de este trabajo- impulsados por la reciente reflexión en torno a la responsabilidad social de los centros universitarios para con la adecuación de programas formativos que respondan a las necesidades reales del mundo laboral, deciden crear una nueva edición de la revista con objetivos distintos, deteniendo la mirada en la alfabetización académica, la literacidad crítica y la multimodalidad de lo digital. En la etapa recientemente arrancada, se decide cambiar parcialmente el título de la revista a Letras de Batalla: debate académico actual. Mantener algo del nombre de la revista es intencional, ya que contribuye a fomentar la marca identitaria de la Universitat. Sin embargo, el cambio de lo que denominaríamos como subtítulo obedece a diversos motivos, tal y como se ha apuntado en la introducción, pero sobre todo a la intención de ampliar el alcance del debate académico suscitado por los artículos publicados en ella con tal de traer el debate académico real a las aulas universitarias y viceversa.

Los objetivos principales de la revista son varios: en primer lugar, incentivar la alfabetización académica de los estudiantes así como su literacidad crítica. Esto se produce estimulando su participación -esto es, animándoles a escribir artículos- y mediante la acción directa de profesores implicados, que ofrecen consejos prácticos para mejorar tanto la forma -lo literal, gramatical...- como el contenido -la lectura entre líneas, la coherencia de los argumentos...- de sus producciones textuales, asimismo impeliéndoles a consultar fuentes científicas con las que contrastar sus argumentos. Estas acciones aspiran, pues, a conformar en el estudiantado una imagen concreta y sólida del mundo y a reafirmar los lazos comunicativos bidireccionales y multimodales que se establecen entre las personas.

El siguiente de los objetivos, en línea con la $3^{\text {a Convocatoria de Proyectos }}$ de Innovación y Mejora de la Calidad Docente convocado por el Vicerrectorado de Ordenación Académica de la Universitat Abat Oliba CEU en la que se presentó este 
proyecto y que fue galardonado con el segundo puesto, se ofrece la posibilidad de utilizar la revista como una herramienta de innovación de la evaluación que permite el uso de la misma como actividad de clase y/o también para premiar el trabajo del alumno excelente y con un nivel de implicación superior a la media, pudiendo ser por ejemplo un instrumento de evaluación para otorgar una matrícula de honor.

En tercer lugar, enriquecer la vida cultural del campus implicando a alumnos de diferentes titulaciones. Esto se realiza fundamentalmente a través de materias transversales como Antropología, Historia de la sociedad, Literatura... etc. Se fomenta también la internacionalización de esta actividad aceptando publicaciones en lenguas extranjeras en todas las secciones.

Asimismo, otro de los objetivos de Letras de Batalla: debate académico actual es incentivar a que los alumnos excelentes se vean motivados a ir más allá de lo obligatorio de la actividad académica y escriban trabajos que supongan una iniciación a la investigación. Esto requiere un seguimiento personalizado del autor -estudiante- de los trabajos por parte de profesorado especializado en las distintas materias que se incluyen en el ámbito de conocimiento de los sucesivos números que se vayan publicando. En lo que respecta a los objetivos relacionados con los estudiantes, se pretende implicar a todos aquellos con capacidad de liderazgo en la gestión de la revista en tanto que miembros de apoyo a los comités de dirección y redacción.

En quinto lugar, la creación de esta conlleva la elaboración de contenidos digitales -se publica online y no en papel- que pueden ser utilizados como herramienta de ampliación y apoyo a la enseñanza presencial en el aula, tanto desde el punto de vista de los contenidos de las asignaturas como de la metodología científica aplicada a los trabajos académicos evaluables.

El sexto de los objetivos es la reimplantación de un premio que ya existía durante la primera etapa de la revista y que dejó de otorgarse por la ausencia de la misma. El Premio Chesterton, vinculado directamente a Letras de Batalla, es convocado por el Vicerrectorado de Estudiantes anualmente a fin de fomentar la implicación del estudiantado en la universidad, y junto con los otros premios y concursos de la Universitat Abat Oliba CEU9 ${ }^{9}$, enriquece la vida académica del campus. Consiste, pues, en galardonar con un diploma acreditativo y una retribución económica de ıoo€ a los tres mejores artículos publicados en el último número de Letras de Batalla. Estos tres mejores artículos son escogidos por una comisión de profesores expertos que evalúan la calidad de los mismos. En cuanto a la suma económica otorgada, los fondos proceden de la financiación recibida por el Vicerrectorado de Ordenación Académica en la convocatoria de los Proyectos Innovación y Mejora de la Calidad Docente. Si bien los gastos financiables estipulados por el Vicerrectorado de Ordenación Académica son aquellos derivados de

\footnotetext{
9 A título informativo, para observar la complejidad de la elección de secciones para la revista en relación a las distintas áreas académicas de la Universitat Ábat Oliba, apuntamos aquí estas últimas: derecho y ciencia política, empresa y economía, comunicación, educación y humanidades, psicología. Dentro de cada área encontramos grados, másteres y posgrados diversos.
} 
la edición e impresión de materiales didácticos y otros materiales fungibles, materiales y libros de consulta, así como la posible formación de los participantes y gastos de desplazamiento, la posibilidad tanto de publicar en formato online la revista como de trabajar conjuntamente entre profesores y estudiantes permite reducir los costos y, por tanto, derivarlos hacia fines que reviertan positivamente en los estudiantes.

A estos objetivos hay que sumarles las correspondientes competencias que el estudiantado desarrolla mediante la participación en la misma y durante la consecución de los objetivos detallados. Por consiguiente, el estudiante se capacitará para adquirir las siguientes competencias:

- Reunir e interpretar datos relevantes para emitir juicios y argumentaciones que incluyan una reflexión sobre temas relevantes de índole social, científica o ética.

- Tener criterios fundados y rigurosos acerca de la sociedad y la cultura actuales.

- Comprender y sintetizar proposiciones complejas, con espíritu crítico, en el contexto en el que se presentan. El logro de esta competencia implica ser capaz de definir, distinguir y relacionar tanto los conceptos básicos como las premisas sobre las que se construye la exposición de cualquier argumento, así como de enunciar y fundamentar su contenido de forma sintética y crítica, en el contexto -científico, político, mediático, organizacional o del tipo que sea- en el que se presentan.

- Transmitir información a un público tanto especializado como no especializado.

- Producir textos escritos académicos en forma de artículo.

- Conocer y poner en práctica distintas estrategias del método de investigación.

- Colaborar con los distintos sectores de la comunidad educativa y del entorno social.

- Trabajar cooperativamente y en equipo hacia la consecución de un objetivo común.

\section{Método}

Las acciones previstas y realizadas para la edición y publicación del número I7 son las que se detallan a continuación. En primer lugar, siguiendo el reglamento interno que ordena la disposición de actividades universitarias de la UAO CEU, se solicita la autorización para el lanzamiento de la revista al Consejo de Gobierno de la Universitat. Habiendo obtenido la autorización, el Comité de Dirección plantea a los Directores de los Estudios, en pequeñas reuniones informales, la posibilidad de participar activamente en ella siguiendo los objetivos previstos; es necesario establecer las secciones de la revista y sus responsables, en otras palabras, aquellos profesores que quieran asumir el papel de jefes de sección y supervisar los artículos recibidos por parte de los estudiantes. Por ello, se tienen en cuenta los distintos grados ofertados y los ámbitos de conocimiento que la revista puede abarcar. Una vez formado el equipo de redacción al completo, y contando con los profesores que muestran interés -entre los que se incluyeron tanto directores de estudios como profesores colaboradores y asociados- y, por tanto, satisfecha la cuota de un jefe de sección por ámbito de conocimiento, tiene lugar una reunión entre el Comité 
de Dirección y el Comité de Redacción. La reunión es crucial para el establecimiento de las secciones definitivas de la revista, de las cuales se designan títulos con los que sus responsables se sienten identificados -afianzando, por consiguiente, su implicación con el proyecto-. Debemos apuntar que no resultó fácil llegar a un acuerdo en la nomenclatura; se reveló la necesidad abarcar la totalidad de los ámbitos de conocimiento ${ }^{\text {IO }}$ de la Universitat para de esa manera abrir a cualquier estudiante la posibilidad de enviar su propuesta en forma de artículo, pero a la vez se acordó, teniendo en cuenta que no todos los profesores de todas las áreas de conocimiento podían implicarse como jefes de sección, que los títulos de las secciones debían ser suficientemente amplios como para que profesores ajenos a la organización de la revista pudiesen recomendarla a sus alumnos e incluso utilizarla como otro instrumento de evaluación más. Finalmente, las secciones dispuestas son: Actualidad editorial, Antropología y filosofía, Ciencias jurídicas, Comunicación y sociedad, Educación, Economía y Empresa, Entrevistas, Historia, Literatura y cine y Persona y salud. Otra de las cuestiones abordadas en este primer encuentro es la participación e implicación de estudiantes en los procesos de organización interna y publicación online de la revista. Se acuerda convocar a todos aquellos interesados en participar en tanto que secretarios de sección para apoyar la labor del responsable de la misma mediante tareas de difusión de la información de la revista entre el alumnado o maquetación del número online, entre otras.

El siguiente paso es difundir públicamente el lanzamiento del próximo número de la revista mediante un comunicado oficial dirigido a toda la comunidad universitaria: se explica el motivo de su reactivación y sus secciones junto a sus responsables. Además, se estipula la fecha límite de envío de artículos para el número en curso. Los medios de difusión de la información proporcionada en este caso contemplan más vías: por un lado, los jefes de sección -en el caso de que no sean la misma persona-informan a los directores de los estudios para que puedan hacer extensiva la invitación a su profesorado mediante el envío de un correo electrónico. En estos correos electrónicos, más personalizados, se incide sobre todo en dos cuestiones: primero, en la posibilidad de servirse de la revista como otra herramienta evaluativa -por ejemplo, proponiendo a todo un grupo la elaboración de un artículo sobre un tema concreto de entre los cuales aquellos mejores tendrán el visto bueno del profesor para que sean enviados a la revista- y segundo, animando a los alumnos a participar en la revista libremente y escribir sobre cualquier tema de su interés para cualquier sección. Por otro lado, en el comunicado también se cita a un día y hora concretos a aquellos alumnos interesados en participar como secretarios de sección. De aquellos que acuden a la mencionada cita y se reúnen con los jefes de sección, una vez definidas y acordadas sus posibles tareas, tres estudiantes asumen esta tarea. La primera de sus acciones es la difundir entre sus compañeros y otros cursos la

\footnotetext{
${ }^{\text {IO }}$ Fruto de esta reunión, se estableció también la sección de “Actualidad editorial”, que no estaba prevista en la planificación de los objetivos de la misma ni responde a ninguna área de conocimiento concreto. Esto es así porque se observó que la actualidad editorial no excluye el debate académico y que la transversalidad es un valor a promocionar, así como la inquietud intelectual de los estudiantes, que pueden no querer verse ceñidos a un ámbito concreto sino a algo que responda más directamente a sus intereses lectores.
} 
convocatoria del número en curso y recordar la fecha de entrega de artículos a la dirección de correo electrónico ${ }^{\text {II }}$ que se crea a propósito de Letras de Batalla. Se considera que es una buena idea -previa aprobación por los directores de estudios, que informan a los profesores que estarían en el aula- que puedan entrar en aulas de primero a cuarto de carrera de las áreas de conocimiento en que ellos se insertan para en cinco minutos hacer una síntesis de la convocatoria abierta para el número en curso.

Tras oficializar el llamamiento para recibir artículos, empieza la fase en la que se recogen los textos y se evalúan por parte de expertos, los jefes de sección. El Comité de Dirección recibe vía email las propuestas de artículos por parte de estudiantes, a las que da acuse de recibo informando, además, que serán sometidas al juicio de los jefes de sección correspondientes. Los encargados de las secciones evalúan los textos mediante una rúbrica que tiene en cuenta los siguientes ítems: a) redacción: argumentación y coherencia b) formato académico: citas, bibliografía c) actualidad: originalidad del tema, impacto social. En base a ese sistema, e imitando el estilo de las revistas académicas, deciden si el texto es apto para su publicación tal y como se ha entregado, o bien si es publicable pero con modificaciones, o bien si el artículo no resulta apropiado para la revista por motivos relacionados con la redacción, formato académico o actualidad. En el caso de que el artículo sea aceptado a condición de aplicar ciertas modificaciones, el jefe de sección se pone en contacto con el autor para que, entre ambos, valoren cómo mejorarlo. El aprendizaje desplegado en esta interacción forma parte de los objetivos didácticos anteriormente explicados: el valor añadido de que un experto indique las carencias de la producción textual y aconseje al estudiante cómo superarlas favorece el aprendizaje significativo y las habilidades para enfrentarse a textos escritos, ya sea escribiéndolos o leyéndolos.

El Comité de Redacción integrado por los jefes de sección se reúne, junto con el Comité de Dirección, aproximadamente dos semanas después de la fecha límite de entrega de los artículos, tiempo suficiente para que cada profesor evalúe cada artículo recibido que corresponda a su sección y trabaje conjuntamente con sus autores. En este encuentro, se ponen en común todos los artículos recibidos, se comparten las valoraciones y las rúbricas evaluativas y finalmente se decide qué artículos saldrán en el próximo número, informando posteriormente de ello vía email a los autores correspondientes. Puede suceder -y ha sucedido así-, que se considere que un artículo interesante exija más tiempo de reelaboración. En ese caso, el jefe de sección correspondiente, aconseja al estudiante seguir trabajando en el mismo y presentarlo al siguiente número.

Tras eso, se procede a planificar la maquetación de la versión online de la revista: es necesario que un profesor asuma la responsabilidad de la tarea y que el estudiantado implicado de manera directa con la revista -según el acuerdo previo establecidoparticipe, recibiendo la formación necesaria por parte de este profesor y acometiendo

\footnotetext{
${ }^{\text {II }}$ El email al que nos referimos, por si los lectores quisiesen más información, es letrasdebatalla@uao.es. Asimismo, la web de la revista es https://letrasdebatalla.uao.es.
} 
aquellas actividades que sus aptitudes, intereses y formación les permitan. Esta tarea puede resultar más o menos compleja en relación a la cantidad de artículos a publicar y de la disponibilidad horaria de los agentes implicados. En la convocatoria del curso 2OI720I8, se publican un total de 16 artículos, de los cuales 4 son artículos de PAS y PDI ${ }^{12}$ y el resto de estudiantes de la universidad, siendo 3 artículos de estudiantes Erasmus, es decir, en inglés. La relación de artículos publicados en secciones ${ }^{13}$ es la que sigue:

- Antropología y Filosofía: I artículo

- Ciencias Jurídicas: 4 artículos

- Comunicación y Sociedad: I artículo

- Educación: 3 artículos

- Entrevistas: I artículo

- Literatura y cine: 4 artículos

- Persona y Salud: 2 artículos

El número de la revista es publicado online y se informa a la comunidad universitaria mediante un comunicado. Pasadas dos semanas aproximadamente, el Comité de Dirección, aconsejado por el Comité de Redacción, procede a reunir un tribunal de expertos y profesores que fallan el Premio Chesterton ${ }^{\mathrm{I} 4}$ en base a las primeras rúbricas de evaluación elaboradas por los jefes de sección y el análisis del artículo publicado. El tribunal se compone tanto de algunos jefes de sección como de profesores de distintos ámbitos de conocimiento que son invitados a formar parte del mismo. Así, se escoge a los tres mejores artículos publicados, teniendo por norma no galardonar a más de un artículo por sección. El Premio Chesterton se concede durante la celebración del día de Santo Tomás de Aquino en la Universitat.

Finalizado este ciclo, se reúnen ambos comités de nuevo para valorar el funcionamiento del proyecto, la organización de las distintas fases y la imbricación entre profesores, estudiantes y comunidad universitaria en la publicación online de la revista. Asimismo, en este encuentro final de ciclo, se pactan de nuevo las fechas correspondientes a cada una de las fases detalladas de cara al curso siguiente, es decir, para el siguiente número de la revista, y las acciones a llevar a cabo.

\footnotetext{
${ }^{\text {I2 }}$ Con tal de animar a toda la comunidad universitaria a participar, el personal de administración y servicios (PAS) así como el personal docente e investigador (PDI) pueden enviar sus propuestas de publicación, dando así ejemplo y apoyo a los estudiantes participantes. No obstante, sus textos no participan del Premio Chesterton.

I3 Como el lector habrá notado, algunas secciones quedan desiertas al no recibir artículos relacionados con esa temática. Con tal de mejorar para la siguiente convocatoria, los Comités de Dirección y Redacción se comprometen a dar más visibilidad a la revista desde esos ámbitos, implicando a más profesores si hace falta.

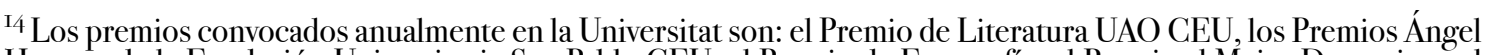
Herrera de la Fundación Universitaria San Pablo CEU, el Premio de Fotografía, el Premio al Mejor Deportista, el concurso promocional de movilidad internacional UAO CEU y el Premio Chesterton.
} 


\section{Resultados}

En este apartado reunimos los datos cuantitativos y cualitativos fruto de la experiencia de innovación expuesta con tal de realizar una valoración final de su utilidad en tanto que metodología docente así como del cumplimiento de sus objetivos iniciales. Así pues, durante el curso 20I7-20I8 se ofreció un espacio de intercambio de conocimiento inicialmente destinado tanto a aquellos estudiantes con intereses extracurriculares como a aquellos con rendimiento académico satisfactorio y que tuviesen un interés especial en mejorar su alfabetización científica. A este ofrecimiento, respondieron doce alumnos presentando cada uno una propuesta de producción científica académica, de los cuales el 40\% contaban con una media académica superior a 7.5. Estos números nos descubren que los destinatarios reales de la experiencia fueron, por tanto, los esperados: un 40\% cubrió un posible vacío en cuanto a la oferta de actividades no formales disponibles y satisfizo su interés en potenciar sus habilidades de lectoescritura textual, mientras que un más amplio 6o\% de los que escribieron un artículo aparentemente lo hicieron motivados por factores como podrían ser la recomendación por parte del profesorado, entre otros, lo cual a su vez revela la utilidad del proyecto como herramienta de apoyo a la evaluación continua en las asignaturas. El seguimiento personalizado de los profesores-especialistas a los estudiantes-autores fue constante y fructífero, como se ha hecho patente al observar la calidad de las producciones: se recoge por parte de estos especialistas que tuvieron que detenerse de modo particular en los encuentros con autores en la consulta de fuentes científicas y en la coherencia de los argumentos expuestos en sus artículos, mientras que, en menor medida, destacan revisiones de forma en lo que respecta a errores de concordancia gramatical. En ambos casos, los profesores especialistas aseguran que la revisión acompañada de los artículos contribuyó a generar y reforzar en los estudiantes una conciencia sobre la multimodalidad comunicativa que se establece en el camino hacia la adquisición de una alfabetización científica óptima, esto es, crítica, responsable y eficiente. Esto conlleva, a su vez, la adquisición en mayor o menor medida de las competencias esbozadas con anterioridad, a saber: la síntesis de cuestiones complejas, el perfeccionamiento de la interpretación de datos con el objetivo de emitir juicios y argumentaciones, la construcción de criterios rigurosos sobre la sociedad actual, la transmisión de información a un público amplio y diverso, la puesta en práctica de estrategias y métodos de investigación, la colaboración con el ámbito educativo y el trabajo cooperativo.

Se intentará mejorar en una segunda fase de implementación la integración de la revista en las guías docentes de las asignaturas cumpliendo así en su totalidad el segundo objetivo inicial: tres de los siete profesores del Comité de Redacción instaron a sus grupos clase a redactar un artículo para la revista como actividad obligatoria, pero esta no fue contemplada por escrito, como se mencionaba, en las correspondientes guías docentes.

Si continuamos con la participación del alumnado, se recibió una cantidad suficiente de artículos como para publicar un número de la revista, tal y como se 
esperaba, aunque no se satisfizo la expectativa de recibir, como mínimo, un artículo por cada sección, de modo que ciertas secciones quedaron desiertas y otras tuvieron más de un artículo. Se recibieron propuestas de artículos de tres estudiantes internacionales, lo cual permitió alcanzar el objetivo que pretendía enriquecer la vida cultural del campus así como el diálogo entre estudiantes de distintas titulaciones y cursos. Este diálogo también aconteció y fue reforzado por los profesores participantes e integrantes de los comités, especialmente el de Dirección, procedentes de distintas áreas de conocimiento, quienes contribuyeron a la mejora de la calidad del proyecto con sus juicios y puntos de vista. Resultó gratificante comprobar el compromiso, la motivación y disponibilidad de profesores de otras áreas con los que poder intercambiar experiencias comunes. Fruto de esta colaboración pudo también cumplirse el sexto objetivo, constituyéndose el tribunal del Premio Chesterton para fallarlo y otorgar así un premio a los tres mejores artículos de distintas secciones. Por otro lado, se contó con la participación de tres estudiantes que apoyaron al Comité de Redacción, doblando así la previsión inicial de contar con solamente con uno. Este apoyo se materializó en el cumplimiento del quinto de los objetivos iniciales, a saber la creación de contenidos digitales, publicando digitalmente la revista.

Ciertamente, en este primer curso de funcionamiento de la revista, el escaso recorrido temporal de la misma en esta nueva etapa impide valorar de manera profunda ciertos ítems sometidos a una evaluación cualitativa, como lo es la mejora de la alfabetización científica, si bien es posible apuntar una mejora significativa en la consecución de este primer objetivo de la experiencia, puesto que la alfabetización académica es también una habilidad que exige práctica. Aunque para ser evaluada requiera de una medición prolongada en el tiempo así como de métodos de triangulación varios, cabe subrayar que la producción de un artículo científico en un contexto que favorece las prácticas letradas académicas no es un asunto trivial sino más bien todo lo contrario: una primera práctica letrada significativa constituye también uno de los cimientos de una sólida alfabetización académica. No obstante, teniendo en cuenta esta limitación, se prevé explorarla próximamente buscando nuevas maneras para registrar el seguimiento de aquellos estudiantes cuya implicación con la revista sea constante y no puntual. Una de las medidas que consideramos oportunas adoptar es la realización de unas entrevistas al inicio y al final del proceso de publicación de un número de la revista, desde que se envía la invitación a enviar artículos hasta que se publican en la web los artículos recibidos y revisados. Estas entrevistas a los estudiantes que envíen sus propuestas pueden ayudar a determinar cuánto autoconocimiento disponen en lo que se refiere a su nivel de literacidad y qué carencias lectoescritoras pueden necesitar compensar mediante actividades complementarias como lo es la revista. Asimismo, las entrevistas finales permitirán identificar y recoger la experiencia de aprendizaje de estos mismos estudiantes durante todo el proceso. 


\section{Conclusiones}

Se ha observado que las habilidades lectoras y de escritura son conocimientos procesuales, es decir, construcciones cognitivas que progresan y crecen en el tiempo y, por tanto, se modifican en base a los varios discursos y contextos (Marín, 20o6: 36). Por consiguiente, en tanto que procesos, pueden ser enseñados, aprendidos y perfeccionados cuanto se quiera. Se ha señalado, también, la existencia de prácticas letradas académicas así como la necesidad de incentivarlas desde el ámbito universitario, que actualmente, parece no disponer de los métodos adecuados para fundamentar la enseñanza de esta alfabetización académica. Esta poca intención en la proyección de la iniciación y el perfeccionamiento de la lectura y producción de lenguaje científico se contradice con las características propias del lenguaje científico per se, que se contrapone a otro tipo de producciones textuales distintas a estas en lo que respecta al ámbito en el que se despliegan. Así pues, es posible distinguir entre lo vernáculo y lo académico: mientras que lo vernáculo se queda en el ámbito privado siendo un aprendizaje informal y hasta cierto punto poco relevante pero muy vinculado a la propia identidad, elámbito académico está regulado e impuesto por instituciones varias, se inserta dentro de la categoría del aprendizaje formal, es socialmente legitimado y se vincula con la capacidad de recibir y comunicar información del exterior, sobre el mundo (Cassany, 2oogb: II).

De ahí se deriva que la experiencia pedagógica de revista universitaria descrita se proponga como un método de aprendizaje no formal -esto es, extracurricular- aunque con posibilidades de vincularse al formal y académico de manera organizada y explícita como método de fomento y práctica de la lectura y escritura académica. Esto promueve el despertar de una conciencia común de esta necesidad, la apertura al diálogo con el mundo entero y la ruptura de tópicos; no es lo mismo saber leer y escribir que leer y escribir con el dominio del conocimiento de una disciplina, y la elaboración del conocimiento pasa precisamente por el discurso escrito (Cassany y Morales, 2009).

En definitiva, gracias a Letras de batalla: debate académico actual, se proporciona un método para alimentar la literacidad crítica de estudiantes universitarios utilizando sus medios y lenguaje. Solo cuando conozcamos las casuísticas varias de las prácticas letradas contemporáneas y las hagamos propias lograremos los resultados que pueden esperarse en esta sociedad postmoderna en relación a la noción de literacidad. 


\section{Referencias bibliográficas}

- Barton, D. y Hamilton, M. (I998). La literacidad entendida como práctica social. En V. Zavala, M. Niño-Murcia, P. Ames (Eds.). Escritura y sociedad: nuevas perspectivas teóricas y etnográficas (pp. Io9-I39). Perú: Red Para el Desarrollo de las Ciencias Sociales en Perú.

- Cassany, D. (2006). Tras las líneas. Sobre lectura contemporánea. Barcelona: Anagrama.

- Cassany, D. (Comp.) (2009). Para serletrados. Voces y miradas sobre la lectura. Barcelona: Paidós Educación.

- Cassany, D. (2009b). Prácticas letradas contemporáneas: claves para su desarrollo. En Congreso Leer. es, Madrid, I5 de novembre de 2003. Madrid: Ministerio de Educación. Obtenido el Io de marzo de 2020 desde https://repositori.upf.edu/bitstream/ handle/IO230/2I294/Cassany_LEERES.pdf

- Cassany, D. (2012). En_línea. Leery escribir en la red. Barcelona: Anagrama.

- Cassany, D. (20I9). Laboratorio lector. Para entender la lectura. Barcelona: Anagrama.

- Cassany, D. y Morales, O.A. (2009). Leer y escribir en la universidad: los géneros científicos. En D. Cassany (Comp.). Para ser letrados. Voces y miradas sobre la lectura (pp. ıo9-I28). Barcelona: Paidós.

- Cassany, D. y Sala, J. (2009). ¿Enseño a leer a mis alumnos de manera crítica? En D. Cassany (Comp.). Para ser letrados. Voces y miradas sobre la lectura (pp. 8I-97). Barcelona: Paidós Educación.

- Cassany, D.; Sala, J. y Hernández, C. (2008). Escribir «al margen de la ley»: prácticas letradas vernáculas de adolescentes catalanes. En A. Moreno Sandoval (Coord.). El valor de la diversidad (meta)lingüística: Actas del VIII congreso de Lingüística General. Obtenido el 27 de marzo de 2020 desde http://www.lllf.uam.es/clg8/actas/index.html

- Kozak Rovero, G. (200I) ¿ ¿Adónde va la literatura? La escritura, la lectura y la crítica entre la galaxia Gütenberg y la galaxia electrónica. Revista Iberoamericana, LXVII(I97), 687-7O7.

- Lankshear, C. y Knoble, M. (2006). New Literacies: Everyday Practices and Classroom Learning. Nueva York: McGraw Hill.

- Lipovetsky, G y Charles, S. (20I4). Los tiempos hipermodernos. Barcelona: Anagrama.

- Marín, M. (2006). Alfabetización académica temprana. Lectura y vida: Revista latinoamericana de lectura, 27(4), 30-39. 
- Prensky, M. (200I). Digital natives, digital immigrants, part I. On the Horizon, 9(5), I-6.

- Reale, G. (2005). La crisis actual de la cultura escrita. En G. Reale. Raíces culturales y espirituales de Europa (pp. I45-I48). Barcelona: Herder.

- Rodríguez, J.A. (20I3). La ciudad post letrada: reto para la escuela contemporánea. Educación y ciudad, 25, 39-50.

- Street, B. (2004). Los nuevos estudios de literacidad. En V. Zavala, M. Niño-Murcia y P. Ames, (Eds.). Escritura y sociedad: nuevas perspectivas teóricas y etnográficas (pp. 8I-IOz). Perú: Red Para el Desarrollo de las Ciencias Sociales en Perú.

- Tang, K.-S. y Danielsson, K. (20I8). The Expanding Development of Literacy Research in Science Education Around the World. En K.-S. Tang y K. Danielsson (Eds.). Global Developments in Literacy Research for Science Education (pp. I-II). Switzerland: Springer.

- Zavala, V. (2008). La literacidad o lo que la gente hace con la lectura y la escritura. Textos de Didáctica de la Lengua y la Literatura, 47, 7I-79.

- Zavala, V. (2018). El lenguaje como práctica social: cuestionando dicotomías y esencialismos en la educación intercultural bilingüe. Sílex, I, 57-76.

-Zavala, V.; Niño-Murcia, M. y Ames, P. (Eds.) (2004). Escritura y sociedad: nuevas perspectivas teóricas y etnográficas. Perú: Red Para el Desarrollo de las Ciencias Sociales en Perú. 